\title{
Configuration Design of an Under-Actuated Robotic Hand Based on Maximum Grasping Space
}

\author{
Shang-Ling Qiao ${ }^{1}$, Rong-Qiang Liư${ }^{1}$ Hong-Wei Guo ${ }^{1 *}$, Yu-Xuan Liu² and Zong-Quan Deng ${ }^{1}$
}

\begin{abstract}
Capture is a key component for on-orbit service and space debris clean. The current research of capture on-orbit focuses on using special capture devices or full-actuated space arms to capture cooperative targets. However, the structures of current capture devices are complex, and both space debris and abandoned spacecraft are noncooperative targets. To capture non-cooperative targets in space, a lightweight, less driven under-actuated robotic hand is proposed in this paper, which composed by tendon-pulley transmission and double-stage mechanisms, and always driven by only one motor in process of closing finger. Because of the expandability, general grasping model is constructed. The equivalent joint driving forces and general grasping force are analyzed based on the model and the principle of virtual work. Which reveal the relationship among tendon driving force, joint driving forces and grasping force. In order to configure the number of knuckles of finger, a new analysis method which takes the maximum grasping space into account, is proposed. Supposing the maximum grasped object is an envelope circle with diameter of $2.5 \mathrm{~m}$. In the condition, a finger grasping maximum envelope circle with different knuckles is modeled. And the finger lengths with corresponding knuckles are calculated out. The finger length which consists of three knuckles is the shortest among under-actuated fingers consists of not more than five knuckles. Finally, the principle prototype and prototype robotic hand which consists of two dingers are designed and assembled. Experiments indicate that the under-actuated robotic hand can satisfy the grasp requirements.
\end{abstract}

Keywords: Under-actuated robotic hand, Tendon-pulley transmission, Grasping space, Configuration design

\section{Introduction}

With the increase of human space activities and deep space exploration missions, the Earth orbit is in a serious predicament caused by millions of space debris [1]. Fragments of defunct spacecraft captured in orbit technology have become an important direction of the development of space technology.

Space robotic arms play an increasingly important role in on-orbit serving missions [2,3]. Many robotic arms adopted full-driven mechanism have been developed and used in space capture [4]. Typical operational targets on orbit are cooperative in the sense that they allow

\footnotetext{
*Correspondence: guohw@hit.edu.cn

1 State Key Laboratory of Robotics and System, Harbin Institute

of Technology, Harbin 150001, China

Full list of author information is available at the end of the article
}

friendly communication for synchronization manipulation, as well as dedicated grappling fixtures and artificial markers to support docking [4]. The capture process of cooperative spacecraft is almost static or quasi-static in low earth orbits, such as the famous CX-OLEV mission [5] and SMART-OLEV spacecraft [6]. Nowadays, the robotic arms with full driven have been widely applied to the tasks in space, such as docking, berthing, refueling, repairing and upgrading [7-9]. However, the structures of those full driven space arms are relatively complex, heavy, and driven by more motors. And the conventional full-driven robotic arms need complex sensor systems. The control systems are relatively complex for griping different shape objectives [10].

Besides, the space debris are generally non-cooperative targets with uncertain patterns and grappling fixtures. Space debris are often out of control and their movement
Springer Open

(C) The Author(s) 2018. This article is distributed under the terms of the Creative Commons Attribution 4.0 International License (http://creativecommons.org/licenses/by/4.0/), which permits unrestricted use, distribution, and reproduction in any medium, provided you give appropriate credit to the original author(s) and the source, provide a link to the Creative Commons license, and indicate if changes were made. 


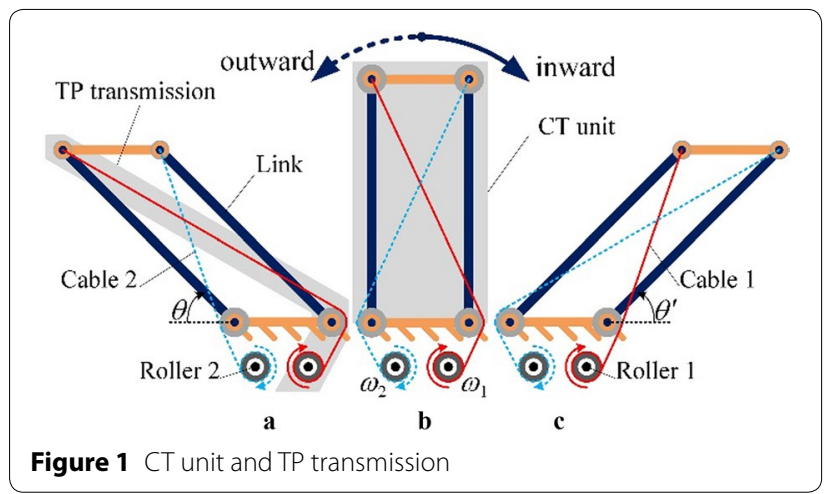

characteristics might not be known beforehand, so they cannot be captured in a stable environment [11]. Hence, much attention has been given to the novel space robotics where the number of control inputs is less than that of degrees of freedom [12, 13]. Such space robotics are named as under-actuated space robotics, which process more unique advantages of light weight, simple structure and well self-adaptation than the full actuated space arms [14]. One famous under-actuated robotic hand, named as SARAH, has three fingers and 10-DOF driven by two motors, which has been used in space station [15].

The principle of under-actuation has been applied to the design of robot hands since the 1970s [16]. Pioneer designs lots of famous under-actuated robotic hands using linkage, tendon and gear transmissions [17-20]. Generally, tendons robotic hands own a good expandability than linkage and gear hands. Columbia hand is a highly underactuated hand containing 3 fingers and every finger with 3-DOF, driven by only two actuators [21]. LISA Hand has 5 fingers and 14 DOFs, which is driven by only 5 motors [22]. Every finger in iHY hand is driven by a single flexor tendon $[23,24]$. The ARM-H hand is made up by four identical fingers which is connected to a main driver board located inside of the palm unit [25]. Through the physiology dissection of human finger, tendons robotic fingers are more similar to human. Two tendons are used to control human finger bending inward and outward [26]. With the development of bionics, the dexterous hands based on characteristics of a human hand have also been developed with tendon transmission $[27,28]$. Tendon-driven mechanisms use flexible cables to deliver the driving force to the joints, and the cables play a similar role as tendons in human hands. Besides, an increasing number of the soft grippers [29-31], dexterous hands [32-35] and parallel manipulators [36] adopt the tendon transmission.

Although many manipulators have the characteristics of under-actuated mechanisms, some shortcomings of complex structures, non-expandable and many drive motors still be there. Even a class of robotic hands which overcome those shortcomings, driven by cable, have been worked out. But those hands are small and not designed for on-orbit grasping large-size object.

In this paper, a novel under-actuated robotic hand driven by tendon is proposed, which combines the linkage and tendon styles. The finger has the characteristics of simple structure, lightweight and less driven. Based on the same maximum grasping space, the grasp model is established. This method is used to analyze the relationship between numbers of segment and lengths of finger. In Section 2, design principle of the finger is introduced. Grasping model, equivalent joint drive and grasping force analysis are carried out in Section 3. Grasp analysis based on enveloping a circular object is introduced in Section 4. According to results of theoretical analysis, the hand consists of two under-actuated finger is designed, and the experimental tests are exhibited in Section 5. Finally, Section 6 concludes this paper (Additional file 1).

\section{Design Principle of the Finger}

The parallel four-linkage mechanism and a cable wrapped along the diagonal lines compose a cable-truss unit (CT unit), shown in Figure 1. Take the red cable as an example, one end of the cable is tied on one side of the diagonal line, the other end of the cable is winded on the roller which is driven by a motor. To transmit the motion, a cable pulley is set up. The cable and cable pulley compose a transmission known as the tendon-pulley transmission (TP transmission).

If several parallel four-linkage mechanisms are assembled in series, and the similar diagonal lines of every parallel four-linkage mechanism is wrapped by the same cable, a novel robotic finger is designed, and every segment of which is a cable-truss unit, illustrated in Figure 2. One cable is used to control the finger bending inward, the other is used to control the finger bending outward. By controlling the tensile force, the motion of the cablebar unit can be controlled.

A finger which owns more than 1-DOF is actuated by a single motor, when the finger bends inward or outward. Thus, the hand belongs to a class of highly under-actuated mechanisms. In a sense, one robotic finger can be driven by only one motor in the grasping process.

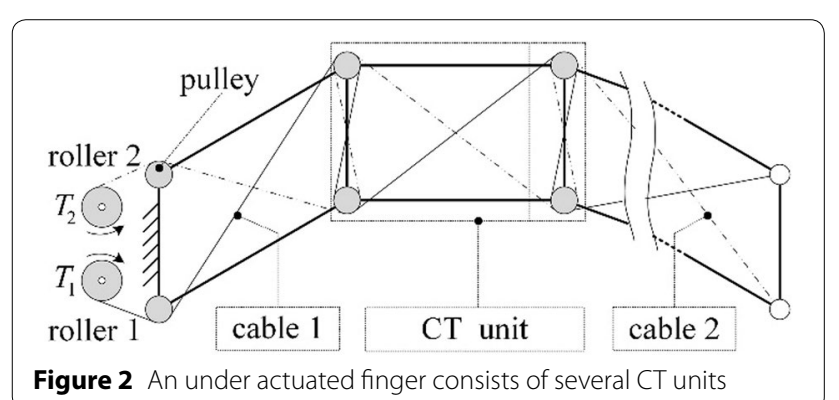




\section{Grasping Model, Equivalent Joint Drive and Grasping Force Analysis}

\subsection{General Grasping Model of One Finger}

According to the geometric characteristics of the parallel four-linkage mechanism, the general configuration and joint coordinate is illustrated in Figure 3.

There are two coordinate systems to describe the configuration of finger, such as the absolute join system $\left\{\theta_{i}\right\}$ and the relative join system $\left\{q_{i}\right\}$. And, $\theta_{i}$ and $q_{i}$ meet the relationship

$$
\left(\begin{array}{c}
q_{1} \\
q_{2} \\
\vdots \\
q_{n-1} \\
q_{n}
\end{array}\right)=\left(\begin{array}{ccccc}
1 & 0 & \cdots & 0 & 0 \\
- & 1 & \cdots & 0 & 0 \\
\vdots & \vdots & \ddots & \vdots & \vdots \\
0 & 0 & \cdots & 1 & 0 \\
0 & 0 & \cdots & -1 & 1
\end{array}\right) \cdot\left(\begin{array}{c}
\theta_{1} \\
\theta_{2} \\
\vdots \\
\theta_{n-1} \\
\theta_{n}
\end{array}\right) .
$$

Although the finger driven by one motor in moving inside stage, every knuckle can move inside. Hence, the driving force provided by real motor can be hypothetically divided into several parts. All the imaginary driving forces are equivalent joint driving forces, which are coupled with each other. The equivalent joint drive model is illustrated in Figure 4. Which is used for analyzing the relationship between grasping forces and the cable driving force.

Through the method of equivalent joint drive, although all the joint driving forces are imaginary, the tendon-pulley transmission can be equivalent to the full joint driven finger.

\subsection{Equivalent of Joint Driving Forces}

The CT unit is basic component of the finger. In order to simplify the analysis and calculation, the radius of the pulley, and the driving force of cable directly effect on joints are ignored. Due to 1 -DOF of unit $i$, supposing a hypothetical driving force ${ }^{i} M_{e 1}$ which is equivalent to

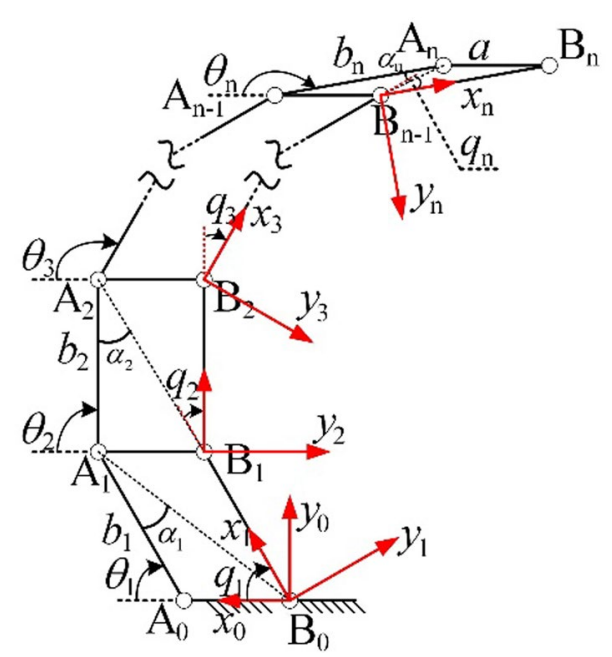

Figure 3 General configuration and joint coordinates

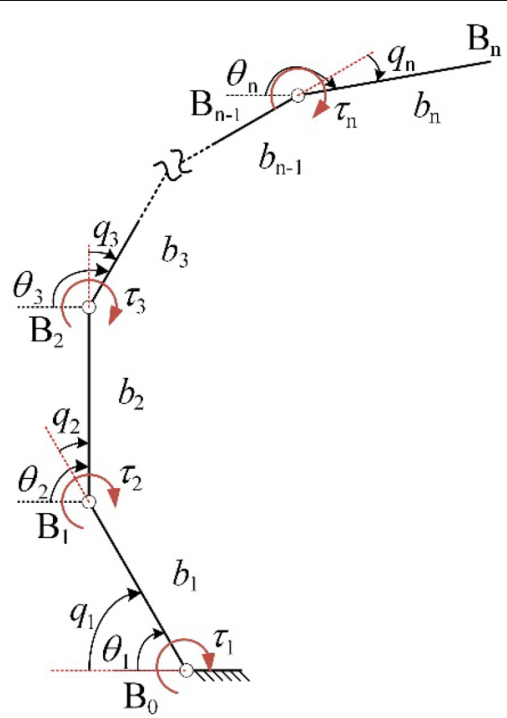

a Equivalent joint drive model

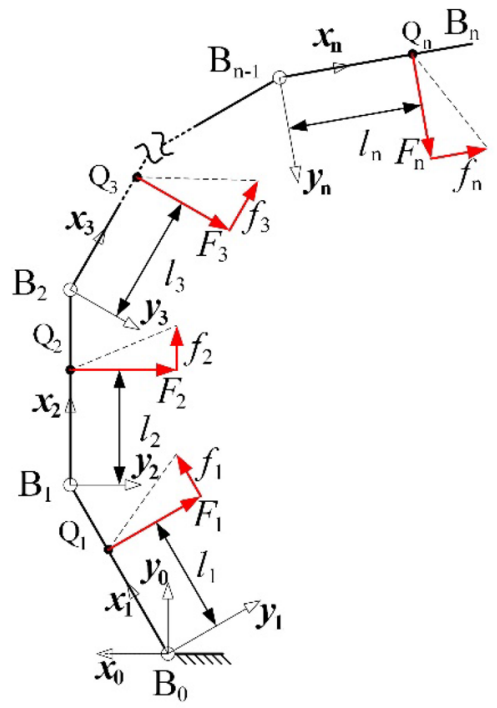

b Grasping forces analysis model

Figure 4 General equivalent model in grasp stage

tendon force $T_{1}$, shown in Figure 5 . Assuming the virtual displacements of cable 1 and the joint are $d^{i} L_{1}$ and $d \theta_{i}$, respectively. And the joint driving force in clockwise direction is " + ". Meanwhile, the analytic method of cable 2 is similar to cable 1 .

According to the virtual work principle, we have

$$
\left\{\begin{array}{l}
d^{i} W_{T 1}=T_{1} \cdot d^{i} L_{1}, \\
d^{i} W_{M_{e 1}}={ }^{i} M_{e 1} \cdot d\left(\pi-\theta_{i}\right), \\
d^{i} W_{T 1}=d^{i} W_{M_{e 1}} .
\end{array}\right.
$$

Thus, 


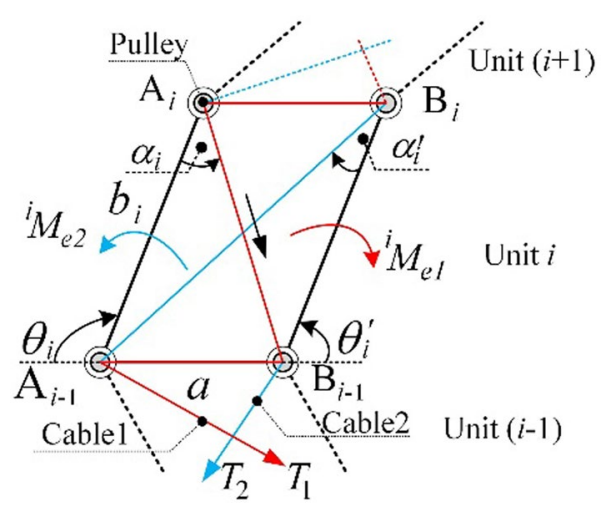

Figure 5 Equivalent model of Joint Driving Force

$$
{ }^{i} M_{e 1}=-T_{1} \cdot \frac{d^{i} L_{1}}{d \theta_{i}} .
$$

Based on rules of simplifications, we have

$$
\begin{aligned}
& { }^{i} L_{1}=\sqrt{a^{2}+b_{i}^{2}+2 a b_{i} \cos \theta_{i}}+a, \\
& \frac{d^{i} L_{1}}{d \theta_{i}}=-\frac{a b_{i} \sin \theta_{i}}{\sqrt{a^{2}+b_{i}^{2}+2 a b \cos \theta_{i}}} .
\end{aligned}
$$

Due to $\sin \alpha_{i}=\frac{a \sin \theta_{i}}{\sqrt{a^{2}+b_{i}^{2}+2 a b \cos \theta_{i}}}$, the conclusion can be
btained,

$$
{ }^{i} M_{e 1}=T_{1} b_{i} \sin \alpha_{i} .
$$

Based on the similar method, for tendon force $T_{2}$ :

$$
{ }^{i} M_{e 2}=T_{2} b_{i} \sin \alpha_{i}^{\prime} .
$$

Thus, the resultant moment of cables in unit $i$ is

$$
{ }^{i} M_{e}=T_{1} b_{i} \sin \alpha_{i}-T_{2} b_{i} \sin \alpha_{i}^{\prime} .
$$

Hypothesizing all joints are driven by motors, shown in Figure 6. Because the resultant moment of cables in unit $i$ is ${ }^{i} M_{e}$, the relationship between $\tau_{i}$ and $\tau_{i+1}$ can be obtained,

$$
\tau_{i}-\tau_{i+1}={ }^{i} M_{e}
$$

Particularly, in distal segment of an $n$-DOF finger, $\tau_{n}$ is equal to ${ }^{n} M_{e}$. Thus, in the $n$-DOF finger, the equivalent joint driving forces can be obtained,

$$
\left(\begin{array}{c}
\tau_{1} \\
\tau_{2} \\
\tau_{3} \\
\vdots \\
\tau_{n-1} \\
\tau_{n}
\end{array}\right)_{n \times 1}=\left(\begin{array}{cccccc}
1 & 1 & 1 & \cdots & 1 & 1 \\
0 & 1 & 1 & \cdots & 1 & 1 \\
0 & 0 & 1 & \cdots & 1 & 1 \\
\vdots & \vdots & \vdots & \ddots & \vdots & \vdots \\
0 & 0 & 0 & \cdots & 1 & 1 \\
0 & 0 & 0 & \cdots & 0 & 1
\end{array}\right)_{n \times n}\left(\begin{array}{c}
{ }^{1} M_{e} \\
{ }^{2} M_{e} \\
{ }^{3} M_{e} \\
\vdots \\
{ }^{n-1} M_{e} \\
{ }^{n} M_{e}
\end{array}\right)_{n \times 1}
$$

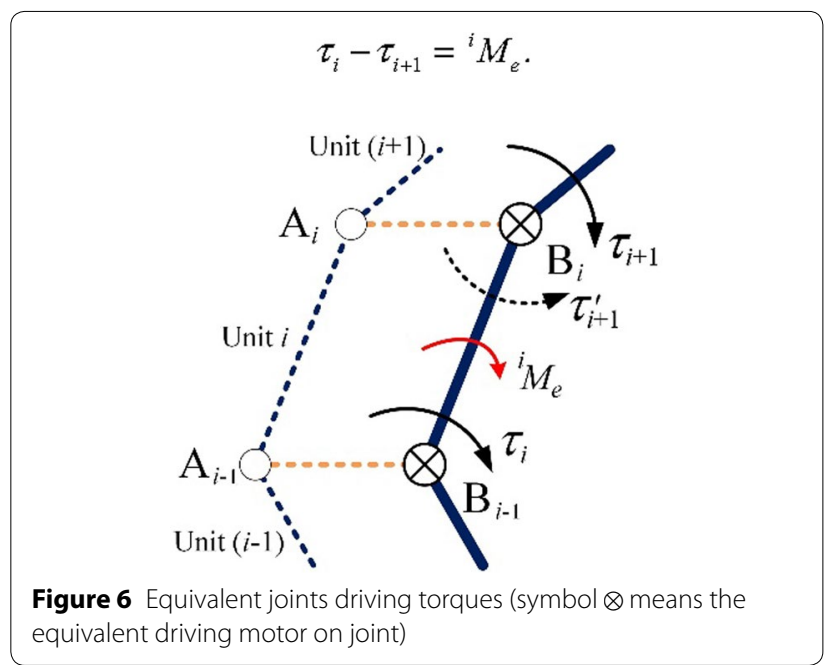

Simply, Eq. (10) could be rewritten as

$$
\boldsymbol{\tau}=\boldsymbol{P}\left(T_{1} \boldsymbol{D}-T_{2} \boldsymbol{D}^{\prime}\right)
$$

where

$\boldsymbol{D}=\left(b_{1} \sin \alpha_{1}, b_{2} \sin \alpha_{2}, \cdots, b_{n-1} \sin \alpha_{n-1}, b_{n} \sin \alpha_{n}\right)^{\mathrm{T}}$,

$\boldsymbol{\tau}=\left(\tau_{1}, \tau_{2}, \cdots, \tau_{n-1}, \tau_{n}\right)^{\mathrm{T}}$,

$\boldsymbol{D}^{\prime}=\left(b_{1} \sin \alpha_{1}^{\prime}, b_{2} \sin \alpha_{2}^{\prime}, \cdots, b_{n-1} \sin \alpha_{n-1}^{\prime}, b_{n} \sin \alpha_{n}^{\prime}\right)^{\mathrm{T}}$.

\subsection{General Grasping Force Analysis}

According to the virtual work principle,

$$
\boldsymbol{\tau}^{\mathrm{T}} \cdot d \boldsymbol{q}={ }^{0} \hat{\boldsymbol{F}}^{\mathrm{T}} \cdot d^{0} \boldsymbol{Q}
$$

Let $\boldsymbol{J}=\frac{d^{0} \boldsymbol{Q}}{d \boldsymbol{q}}$, we have

$$
\boldsymbol{\tau}=\boldsymbol{J}^{\mathrm{T}} \cdot{ }^{0} \hat{\boldsymbol{F}}
$$

where $\boldsymbol{\tau}=\left(\tau_{1}, \tau_{2}, \cdots, \tau_{n}\right)^{\mathrm{T}},{ }^{0} \hat{\boldsymbol{F}}=\left({ }^{0} \hat{\boldsymbol{F}}_{1},{ }^{0} \hat{\boldsymbol{F}}_{2}, \cdots,{ }^{0} \hat{\boldsymbol{F}}_{n}\right)^{\mathrm{T}}$, $\boldsymbol{q}=\left(q_{1}, q_{2}, \cdots, q_{n}\right)^{\mathrm{T}}, \quad{ }^{0} \boldsymbol{Q}=\left({ }^{0} \boldsymbol{Q}_{1},{ }^{0} \boldsymbol{Q}_{2}, \cdots,{ }^{0} \boldsymbol{Q}_{n}\right)^{\mathrm{T}}$, ${ }^{0} \boldsymbol{Q}_{i}=\left(Q_{i x}, Q_{i y}, Q_{i z}\right)^{\mathrm{T}}, \boldsymbol{J}=\left(\boldsymbol{J}_{1}, \boldsymbol{J}_{2}, \cdots, \boldsymbol{J}_{n}\right)^{\mathrm{T}}$.

Due to ${ }^{i} \hat{\boldsymbol{F}}_{i}=\left(f_{i}, F_{i}, 0\right)^{\mathrm{T}},{ }_{i} \boldsymbol{F}_{i}=\left(0, F_{i}, 0\right)^{\mathrm{T}}, \boldsymbol{f}_{i}=\left(f_{i}, 0,0\right)^{\mathrm{T}}$, $F_{i}=\mu_{i} f_{i}$, we obtain,

$$
\boldsymbol{\tau}=\boldsymbol{J}^{\mathrm{T}} \cdot \boldsymbol{R} \cdot \hat{\boldsymbol{F}}=\boldsymbol{J}^{\mathrm{T}} \cdot \boldsymbol{R} \cdot \boldsymbol{\mu} \cdot \boldsymbol{F}_{n o r}
$$

where

$$
\begin{aligned}
& \boldsymbol{\tau}=\left(\tau_{1}, \tau_{2}, \cdots, \tau_{n}\right)^{\mathrm{T}}, \quad \boldsymbol{R}=\operatorname{diag}\left({ }^{0} \boldsymbol{R}_{1},{ }^{0} \boldsymbol{R}_{2}, \cdots,{ }^{0} \boldsymbol{R}_{n}\right), \\
& \boldsymbol{\mu}=\operatorname{diag}\left(\boldsymbol{\mu}_{1}, \boldsymbol{\mu}_{2}, \cdots, \boldsymbol{\mu}_{n}\right), \\
& \boldsymbol{\mu}_{i}=\left(\mu_{i}, 1,0\right)^{\mathrm{T}}, \\
& { }^{i-1} \boldsymbol{R}_{i}=\left(\begin{array}{ccc}
\cos \left(q_{i}\right) & -\sin \left(q_{i}\right) & 0 \\
\sin \left(q_{i}\right) & \cos \left(q_{i}\right) & 0 \\
0 & 0 & 1
\end{array}\right) .
\end{aligned}
$$

Let $\boldsymbol{J}^{*}=\boldsymbol{J}^{\mathrm{T}} \cdot \boldsymbol{R} \cdot \boldsymbol{\mu}, \boldsymbol{J}^{*}$ can be written as 


$$
\boldsymbol{J}^{*}=\left(\boldsymbol{J}_{1}^{\mathrm{T} 0} \boldsymbol{R}_{1} \boldsymbol{\mu}_{1}, \boldsymbol{J}_{2}^{\mathrm{T} 0} \boldsymbol{R}_{2} \boldsymbol{\mu}_{2}, \cdots, \boldsymbol{J}_{n}^{\mathrm{T} 0} \boldsymbol{R}_{n} \boldsymbol{\mu}_{n}\right)
$$

And, Eq. (14) can be simplified as

$$
\tau=J^{*} \cdot F^{*} .
$$

Eq. (16) is the general equation that provides a practical relationship between the driving forces and the grasping forces. According to Eq. (15), let $\boldsymbol{J}_{i}^{*}=\boldsymbol{J}_{i}^{\mathrm{T} 0} \boldsymbol{R}_{i} \boldsymbol{\mu}_{i}, \boldsymbol{J}^{*}$ can be rewritten as $\boldsymbol{J}^{*}=\left(\boldsymbol{J}_{1}^{*}, \boldsymbol{J}_{2}^{*}, \cdots, \boldsymbol{J}_{n}^{*}\right)$.

\section{Grasp Analysis Based on Enveloping a Circular Object}

A finger which consists of several knuckles, grasping a circular object in enveloping form, is shown in Figure 7. Figure 7 (a) shows the grasping configuration of one finger enveloping a circular object and Figure 7 (b) shows the grasping forces on knuckles. $f_{1}, f_{2}$ and $f_{n}\left(f_{i}=\mu_{i} F_{i}\right)$ refer to the frictional forces between knuckles and object, which are tangential component forces of the grasping forces.

According the grasping configuration of one finger enveloping a circular object, the grasping locations of knuckles can be described as

$$
l_{i}=R \tan \frac{q_{i}}{2} .
$$

Hence, one can obtain the lengths of knuckles,

$$
b_{i}= \begin{cases}R\left(\tan \frac{q_{i}}{2}+\tan \frac{q_{i+1}}{2}\right), & i<n, \\ R \tan \frac{q_{i}}{2}, & i=n .\end{cases}
$$

And the installed distance $D$ is

$$
D=R \tan \frac{q_{1}}{2} .
$$

According to Eq. (15), the general grasping forces of enveloping a circular object can be obtained,

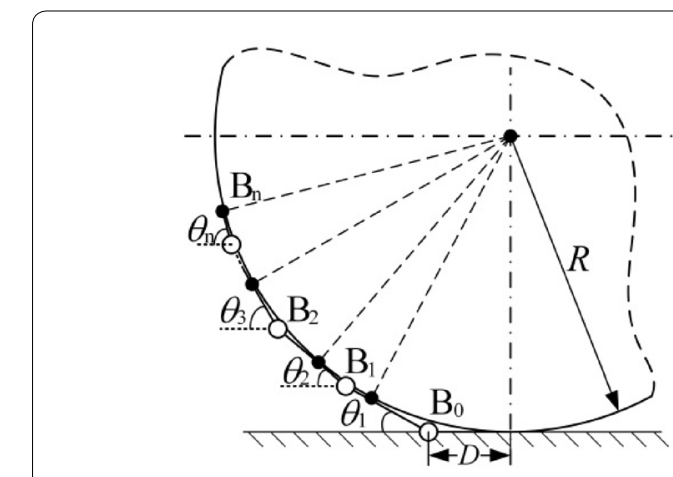

a One finger envelops a circular object

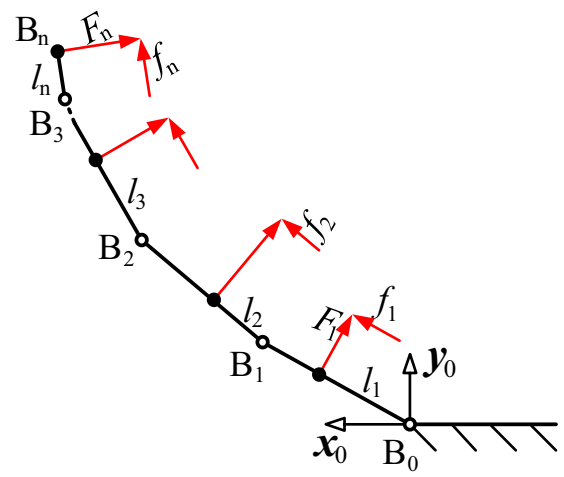

b Grasping forces on knuckles

Figure 7 Configuration of a finger in maximum grasping space

$$
\begin{aligned}
& \left(\begin{array}{cccc}
l_{1} & b_{1} C_{-} q_{2}+\mu_{2} b_{1} S_{-} q_{2} & \cdots & b_{1} C_{-} q_{2,3, \ldots, n}+\mu_{n} b_{1} S_{-} q_{2,3, \ldots, n} \\
0 & l_{2} & \cdots & b_{2} C_{-} q_{3,4, \ldots, n}+\mu_{n} b_{2} S_{-} q_{3,4, \ldots, n} \\
\vdots & \vdots & \ddots & \vdots \\
0 & 0 & \cdots & l_{n}
\end{array}\right) . \\
& \left(\begin{array}{c}
F_{1} \\
F_{2} \\
\vdots \\
F_{n}
\end{array}\right)=T_{1}\left(\begin{array}{c}
b_{1} S_{-} \alpha_{1} \\
b_{2} S_{-} \alpha_{2} \\
\vdots \\
b_{n} S_{-} \alpha_{n}
\end{array}\right) .
\end{aligned}
$$

Sum of the component of grasping forces in $y$-axis direction can be described as
When the finger is in stable grasping state, the condition of steady grasping state can be described as $F_{y} \leq 0$. 
Table 1 Parameters list

\begin{tabular}{llllll}
\hline Parameter & $\boldsymbol{a}(\mathrm{mm})$ & $\boldsymbol{R}(\mathrm{mm})$ & $\boldsymbol{T}_{\mathbf{1}}(\mathbf{N})$ & $\boldsymbol{\mu}$ & $\boldsymbol{q}_{\boldsymbol{i}}$ \\
\hline Value & 100.00 & 1250 & 100 & -0.5 & $(0, \pi)$
\end{tabular}

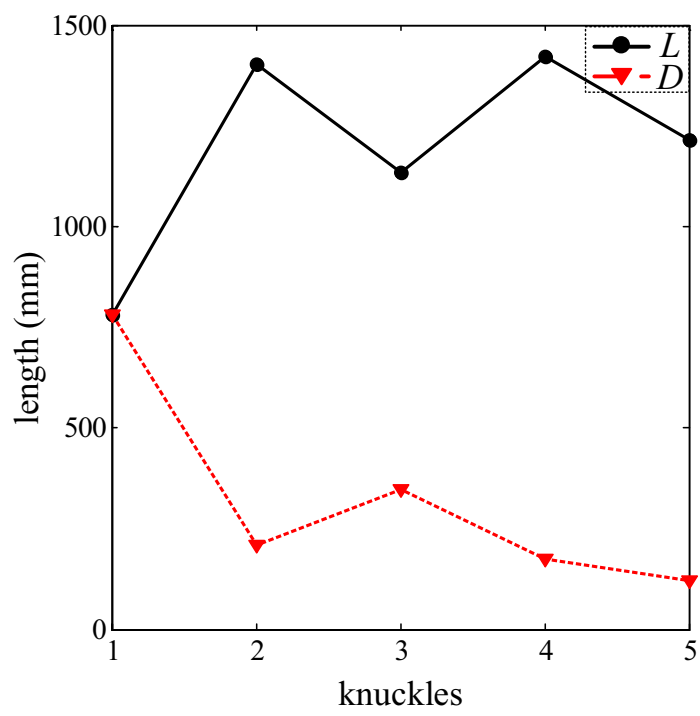

Figure 8 Relationship between configuration and knuckles

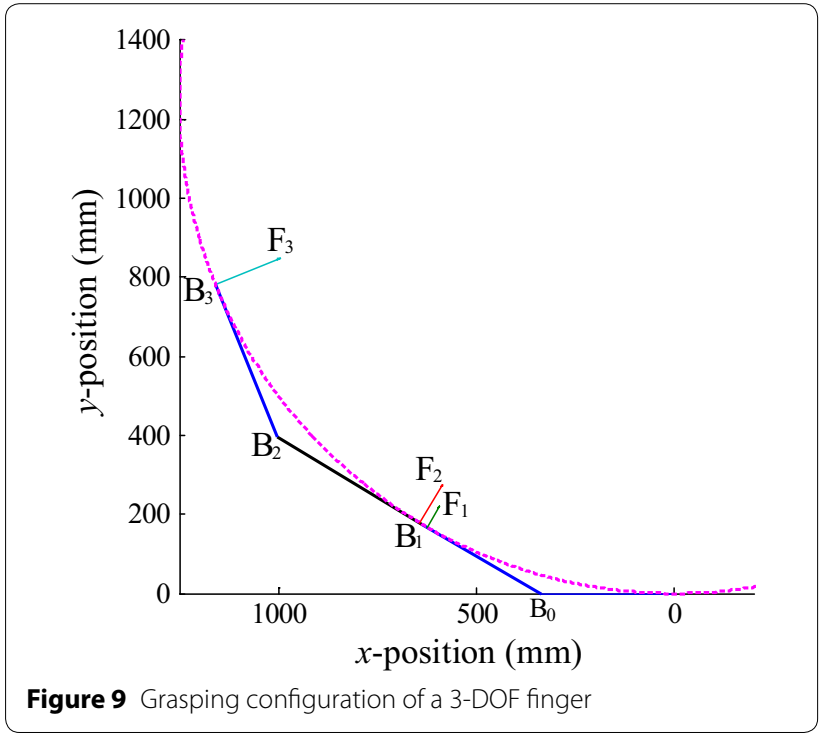

The maximum grasping space is an envelope circle with the diameter of $2.5 \mathrm{~m}$. Due to the maximum grasping space, the object has a tendency of moving outside of finger. Thus, the frictional force on every surface of segment is opposite to the forces on model. Hence, the friction coefficient is negative, and the skin-friction coefficient
Table 2 3-knuckle finger in shortest length finger grasp

\begin{tabular}{lllll}
\hline Parameter & $L(\mathrm{~mm})$ & $D(\mathrm{~mm})$ & $b_{1}(\mathrm{~mm})$ & $b_{2}(\mathrm{~mm})$ \\
Value & 1132.79 & 346.66 & 357.56 & 393.07 \\
Parameter & $b_{3}(\mathrm{~mm})$ & $\theta_{1}\left(^{\circ}\right)$ & $\theta_{2}\left(^{\circ}\right)$ & $\theta_{3}\left(^{\circ}\right)$ \\
Value & 382.16 & 31.00 & 32.01 & 68.02 \\
Parameter & $F_{y}(\mathrm{~N})$ & $F_{1}(\mathrm{~N})$ & $F_{2}(\mathrm{~N})$ & $F_{3}(\mathrm{~N})$ \\
Value & -1.50 & 0.61 & 0.32 & 21.12 \\
\hline
\end{tabular}

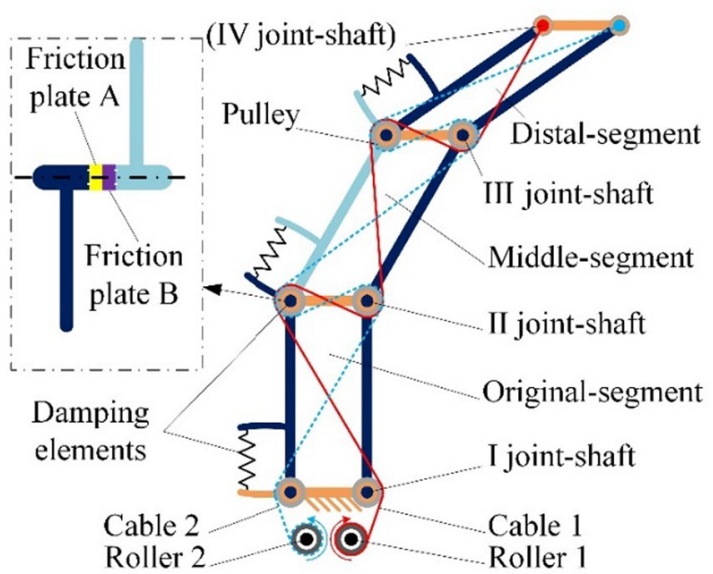

Figure 10 Schematic diagram

of the finger is set as $\mu_{1}=\mu_{2}=\mu_{3}=-0.5$ in grasping maximum object.

Through the numerical analysis, the shortest length of finger, the corresponding length of each knuckle and the configuration of the finger can be obtained. The values of parameters used in calculation are shown in Table 1.

Figure 8 shows the relationship between the shortest finger length and numbers of knuckles. It is a full-actuated finger with the finger owning 1 knuckle. Owing to geometrical feature, the installed distance $D$ is equal to length of the finger. Thus, 1-DOF finger is out of consideration in this paper. With the increase of knuckles, the under-actuated level becomes high. The shortest finger length with three knuckles is the shortest among 2-, 3-, $4-$, and 5-knuckle. But the installed distance is a little big. Although the shortest lengths in 3-, and 5-knuckle are not quite different, the whole weight in 5-knuckle finger is heavier than 3-knuckle finger. Hence, the finger used in experiments is designed with 3-knuckle, and the enveloping grasp configuration with shortest length is shown in Figure 9. The configuration and grasping forces on every knuckle is shown in Table 2. Compared with $F_{3}, F_{1}$ and $F_{2}$ are quite small. But, they are enough for the space applications. Because of one driving force, those component grasping forces are coupled with each other. 

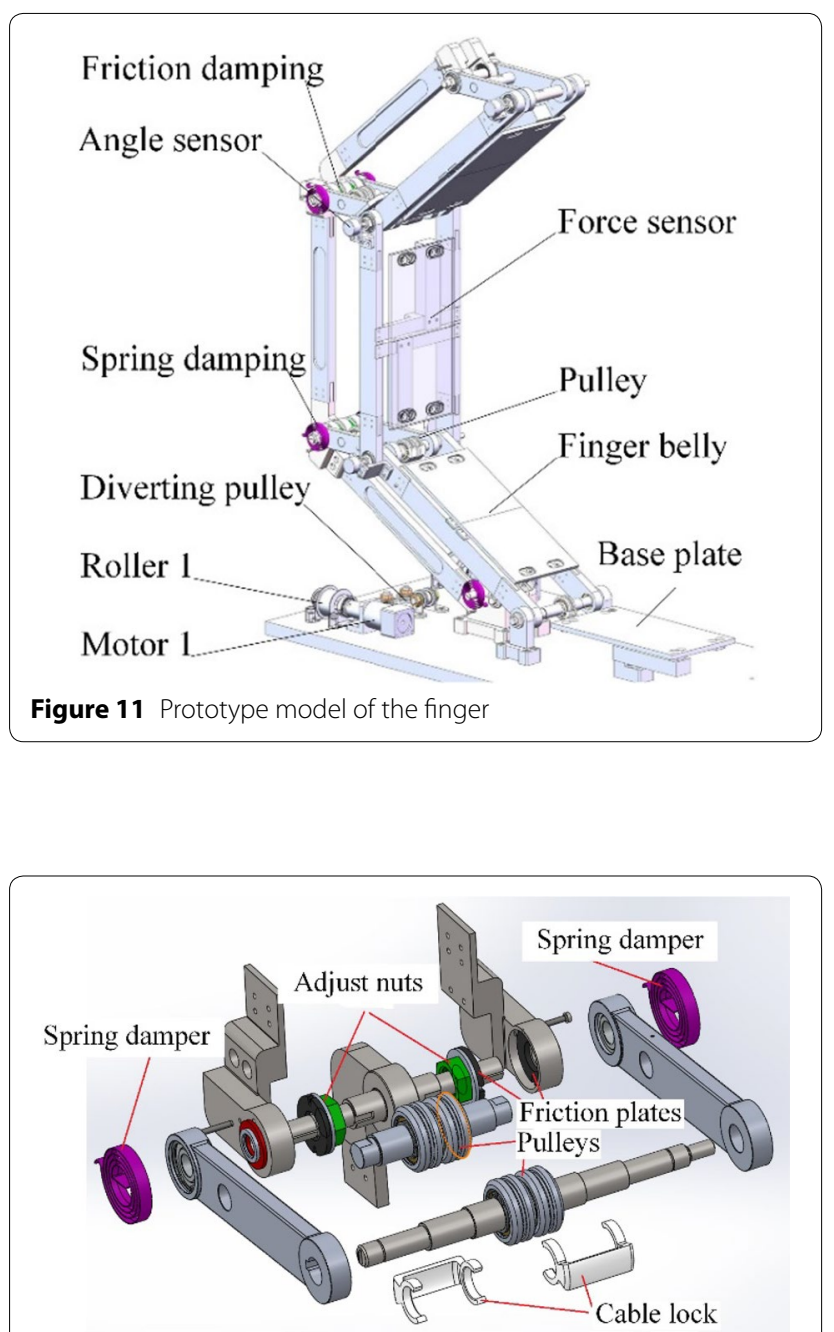

Figure 12 Design of the joint-shaft plane

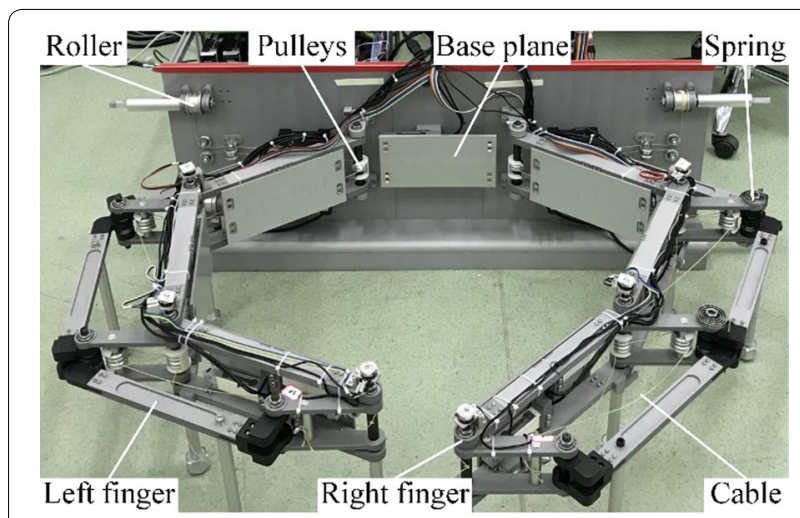

Figure 13 Prototype robotic hand

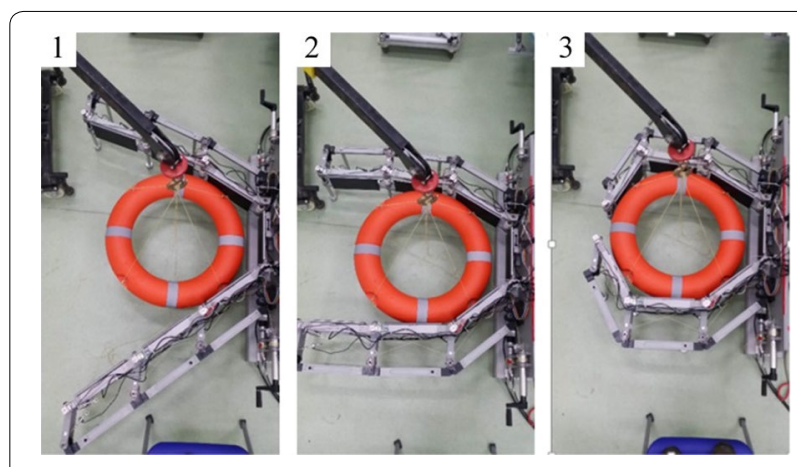

Figure 14 Processes of grasping round object

\section{Design of Under-Actuated Robotic Hand 5.1 Principle Prototype Model}

Here is a schematic diagram of one finger, which contains three knuckles, as shown in Figure 10. With the moving of roller 1 in a certain speed, every knuckle moves inside. Although the finger has 3-DOF, it is strictly driven by only one motor in one moving direction. Figure 11 shows the corresponding prototype model.

In order to control the finger grasping accurately, there are two cable driving force sensors, three joint sensors and two grasping force sensors on each knuckle. Besides, two grasping force sensors are installed on base plate. As the damping elements set on the joint-shafts, the finger owns a certain movement sequence of segments called grasping strategy. The spring damping and friction damping are independent.

Because two kinds of damping elements are set on finger, the sub-planes between knuckle and knuckle are quite complex. Figure 12 shows one joint-shaft plane. The friction damping, consists of two friction plates, can be changed through adjusting adjust nuts.

\subsection{Prototype Robotic Hand and the Experimental Test}

The prototype robotic hand contains two finger, and each finger composed of three knuckles, shown in Figure 13. And there are several experiments to verify the effect in grasps (Additional files 2, 3).

In Figure 14, it is a grasping processes in grasping round object. And it is prepare grasping stage in the first picture. Grasp closing stage is shown in the second picture. Grasping over stage is shown in the third picture.

As a usual, there are two typical grasping over stages in grasping round object. Such as grasp over with wholeknuckle in Figure 15 and grasp over with last two knuckles in Figure 16. As the hand grasping rectangular object, one finger is in grasp over stage, the other is in grasp closing stage, shown in Figure 17. When both finger reach a static equilibrium, the grasping-over stage is realized. 


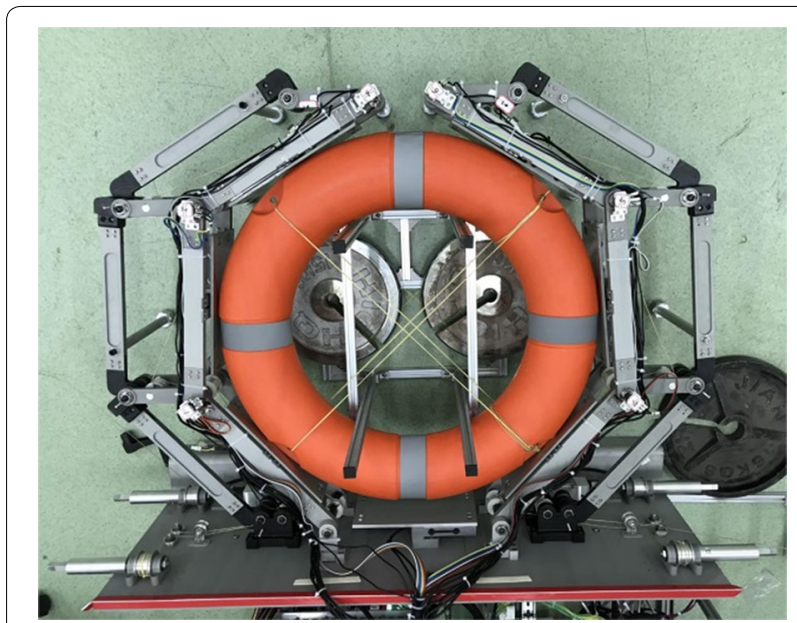

Figure 15 Grasp round object with whole-knuckle

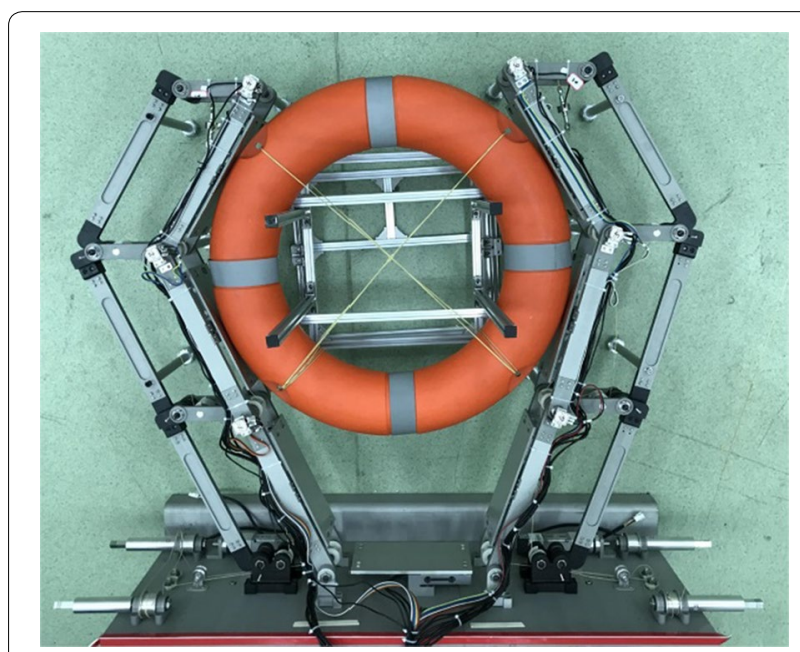

Figure 16 Grasp round object with two-knuckle

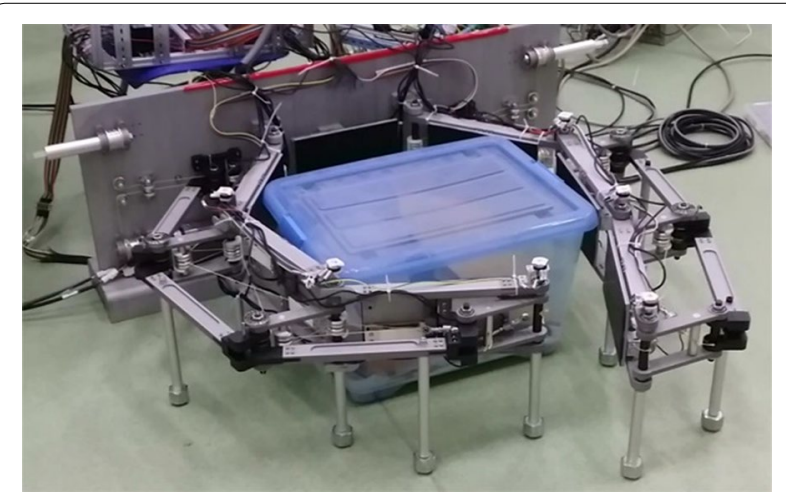

Figure 17 Grasp rectangular object

\section{Conclusions}

In this paper, a novel under-actuated robotic finger composing of cable-truss units was proposed. No matter how many knuckles one finger has, the finger bends inward or outward with two motors. One motor was used to open finger bending outward, the other was used to close finger bending inward. Due to the expandability, general grasping model was constructed. According to the analysis of equivalent joint driving torques and general grasping force, the relationship among cable driving force, joint driving forces and grasping force were revealed. A new analysis method which taken the maximum grasping space into account, was proposed to determine knuckles a finger should own. Supposing the maximum grasped object was an envelope circle with diameter of $2.5 \mathrm{~m}$. A finger composed by different knuckles was modeled in grasping maximum envelope circle. Finger consisted of three knuckles was chosen to design principle prototype model. According to experimental tests, the novel robotic hand was effective in grasps.

Future work includes the characteristics of self-adaptive grasp and the analysis of dynamics for controlling the robotic finger.

\section{Additional Files}

Additional file 1. Brief introduction of the paper

Additional file 2. GIF image of Figure 17.

Additional file 3. GIF image of Figure 14.

\section{Authors' Contributions}

R-QL and H-WG in charge of the whole trial; S-LQ wrote the manuscript; Y-XL and Z-QD assisted with sampling and laboratory analysis. All authors have read and approved the final manuscript.

\section{Author details}

${ }^{1}$ State Key Laboratory of Robotics and System, Harbin Institute of Technology, Harbin 150001, China. ${ }^{2}$ College of International Cooperative Education, Harbin Engineering University, Harbin 150001, China.

\section{Authors' Information}

Shang-Ling Qiao, born in 1990, is currently a PhD candidate at State Key Laboratory of Robotics and System, Harbin Institute of Technology, China. His research interests include under-actuated robotics and under-actuated control. E-mail: qiaosl_hit@163.com.

Rong-Qiang Liu, born in 1965, is currently a professor at Harbin Institute of Technology, China. He received his PhD degree from Harbin Institute of Technology, China, in 1995. His main research interests include wearing robot, military civil power robot and large deployable structure research. E-mail: liurq@hit. edu.cn.

Hong-Wei Guo, born in 1980, is currently an associate professor at Harbin Institute of Technology, China. He received his PhD degree from Harbin Institute of Technology, China, in 2009. His main research interest includes space manipulator system vibration control, large deployable structure and energy absorber optimization. E-mail: guohw@hit.edu.cn.

Yu-Xuan Liu, born in 1997, is currently an undergraduate student at College of International Cooperative Education, Harbin Engineering University, China. E-mail:1870471549@qq.com.

Zong-Quan Deng, born in 1956, is currently a professor at Harbin Institute of Technology, China. His main research interest includes aerospace 
mechanism and control, crashworthiness design of buffer structures, dynamic of robots, the technology of advanced robot. E-mail: dengzq@hit.edu.cn.

\section{Competing Interests}

The authors declare no competing financial interests.

\section{Ethics Approval and Consent to Participate}

Not applicable.

\section{Funding}

Supported by Joint Funds of National Natural Science Foundation of China (Grant No. U1613201), and Shenzhen Research Funds (JCYJ20170413104438332).

\section{Publisher's Note}

Springer Nature remains neutral with regard to jurisdictional claims in published maps and institutional affiliations.

Received: 26 June 2017 Accepted: 17 April 2018

Published online: 06 May 2018

\section{References}

[1] M H Shan, J Guo, E Gill. Review and comparison of active space debris capturing and removal methods. Progress in Aerospace Sciences, 2016, 80: 18-32.

[2] G Genta, M Dolci. Robotic gripper for payload capture in low earth orbit. Proceedings of the ASME International Mechanical Engineering Congress and Exposition, 2016: V04AT05A063-V04AT05A063.

[3] F Feng, YW Liu, H Liu, et al. Design schemes and comparison research of the end-effector of large space manipulator. Chinese Journal of Mechanical Engineering, 2012, 25(4): 674-687.

[4] A Flores-Abad, O Ma, K Pham, et al. A review of space robotics technologies for on-orbit servicing. Progress in Aerospace Sciences, 2014, 68: 1-26.

[5] L Tarabini, J Gil, F Gandia, et al. Ground guided CX-OLEV rendez-vous with uncooperative geostationary satellite. Acta Astronautica, 2007, 61 (1-6): 312-325.

[6] C Kaiser, F Sjoberg, J M Delcura, et al. SMART-OLEV—An orbital life extension vehicle for servicing commercial spacecrafts in GEO. Acta Astronautica, 2008, 63(1-4): 400-410.

[7] G Fantoni, M Santochi, G Dini, et al. Grasping devices and methods in automated production processes. CIRP Annals-Manufacturing Technology, 2014, 63(2): 679-701.

[8] M H Shan, J Guo, E Gill, Review and comparison of active space debris capturing and removal methods. Progress in Aerospace Sciences, 2016, 80: 18-32.

[9] A Krolikowski, E David. Commercial on-orbit satellite servicing: national and international policy considerations raised by industry proposals. New Space, 2013, 1(1): 29-41.

[10] T A Mulder. Orbital express autonomous rendezvous and capture flight operations. Air Force T\&E Days, 2008.

[11] P Huang, J Cai, Z Meng, et al. Novel method of monocular real-time feature point tracking for tethered space robots. Journal of Aerospace Engineering, 2014, 27(6): 04014039.

[12] M A Saliba, CW de Silva, Quasi-dynamic analysis, design optimization, and evaluation of a two-finger underactuated hand. Mechatronics, 2016, 33: 93-107.

[13] S Hettrich, L Napier, C V Felix, et al. The importance of analog planetary research for success and safety of human and robotic space missions. In Space Safety is No Accident. Springer, 2015: 285-293.

[14] T Laliberte, C M Gosselin. Simulation and design of underactuated mechanical hands. Mechanism and Machine Theory, 1998, 33(1-2): 39-57.
[15] C M Gosselin, T Laliberte. Underactuated mechanical finger with return actuation: US, 5,762,390

[16] S Hirose, Y Umetani. The development of soft gripper for the versatile robot hand. Mechanism and Machine Theory, 1978, 13(3): 351-359.

[17] S Yao. Design considerations for an underactuated robotic finger mechanism. Chinese Journal of Mechanical Engineering, 2009, 22(4): 475.

[18] W Z Zhang, D M Che, Q Chen, et al. Development of gesture-changeable under-actuated humanoid robotic finger. Chinese Journal of Mechanical Engineering, 2010, 23(2): 142-148.

[19] C Luo, S C Yang, W Z Zhang, et al. MPJ hand: A self-adaptive underactuated hand with flexible fingers of multiple passive joints. IEEE ICARM 2016-2016 International Conference on Advanced Robotics and Mechatronics (ICARM), 2016: 184-189.

[20] Q Wang, Q Quan, Z Deng, et al. An underactuated robotic arm based on differential gears for capturing moving targets: Analysis and design. Journal of Mechanisms and Robotics, 2016, 8(4): 041012.

[21] L Wang, J DelPreto, S Bhattacharyya, et al. A highly-underactuated robotic hand with force and joint angle sensors. 2011 IEEE/RSJ International Conference on Intelligent Robots and Systems, 2011: 1380-1385.

[22] J Jin, W Z Zhang, Z G Sun, et al. LISA hand: Indirect self-adaptive robotic hand for robust grasping and simplicity. 2012 IEEE International Conference on Robotics and Biomimetics (ROBIO 2012), 2012.

[23] L U Odhner, L P Jentoft, M R Claffee, et al. A compliant, underactuated hand for robust manipulation. The International Journal of Robotics Research, 2014, 33(5): 736-752.

[24] L U Odhner, A M Dollar. Stable, open-loop precision manipulation with underactuated hands. The International Journal of Robotics Research, 2015, 34(11): 1347-1360.

[25] D M Aukes, B Heyneman, J Ulmen, et al. Design and testing of a selectively compliant underactuated hand. The International Journal of Robotics Research, 2014, 33(5): 721-735.

[26] K Dermitzakis, M R Morales, A Schweizer. Modeling the frictional interaction in the tendon-pulley system of the human finger for use in robotics. Artif. Life, 2013, 19(1): 149-169.

[27] C Rossi, S Savino. An underactuated multi-finger grasping device. International Journal of Advanced Robotic Systems, 2014, 11(2): 20.

[28] R Carpenter, R Hatton, R Balasubramanian. Evaluation of linear and revolute underactuated grippers for steel foundry operations. Industrial Robot: An International Journal, 2015, 42(4): 314-323.

[29] J Gafford, Y Ding, A Harris, et al. Shape deposition manufacturing of a soft, atraumatic, and deployable surgical grasper. Journal of Mechanisms and Robotics-Transactions of the ASME, 2015, 7(2): 021006.

[30] A Firouzeh, J Paik. Grasp mode and compliance control of an underactuated origami gripper using adjustable stiffness joints. IEEE-ASME Transactions on Mechatronics, 2017, 22(5): 2165-2173.

[31] L Barbazza, D O Zanotto, G Rosati, et al. Design and optimal control of an underactuated cable-driven micro-macro robot. IEEE Robotics and Automation Letters, 2017, 2(2): 896-903.

[32] B Peerdeman, M Valori, D Brouwer, et al. UT hand I: A lock-based underactuated hand prosthesis. Mechanism and Machine Theory, 2014, 78: 307-323.

[33] G Carbone, C Rossi, S Savino. Performance comparison between FEDERICA hand and LARM hand. International Journal of Advanced Robotic Systems, 2015, 12(7): 90.

[34] C Rossi, S Savino, V Niola, et al. A study of a robotic hand with tendon driven fingers. Robotica, 2014, 33(5): 1034-1048.

[35] R Ozawa, K Hashirii, Y Yoshimura, et al. Design and control of a threefingered tendon-driven robotic hand with active and passive tendons. Autonomous Robots, 2014, 36(1-2): 67-78.

[36] B Ouyang, W W Shang. Rapid optimization of tension distribution for cable-driven parallel manipulators with redundant cables. Chinese Journal of Mechanical Engineering, 2016, 29(2): 231-238. 